\title{
Watermarking Digital Artworks
}

\author{
W.K. EISaid \\ Lecturer in Computer Science \\ Mansoura University, Egypt
}

\begin{abstract}
This paper presents a digital watermarking scheme based on a new application of DWT and SVD for color artworks protection. This scheme uses the major features of the two chrominance components where the watermark bits are embedded only into one chrominance component according to the color prevalence rate. In watermark embedding process, more modifications can be done upon the selected chrominance component, while more readable information is needed during the watermark extraction process. Simulation results demonstrate that the proposed scheme yields a high level of robustness against various image processing operations without sacrificing the visual quality of watermarked artwork image.
\end{abstract}

\section{Keywords}

Digital Watermarking, Artworks, Color Prevalence Rate, DWT, SVD.

\section{INTRODUCTION}

The modern technological advancement in the field of multimedia content distribution has great positive effects on a major reorganization of this trade. One of these effects is that valuable digital artworks can be reproduced and distributed arbitrarily without the control of copyright holders. However, the digital representation of multimedia content has some drawbacks such as copyright piracy, an illegal copy and editing. Therefore, the protection and management of intellectual property rights in digital world has become an essential issue[1].Many security solutions have been proposed in the literature to protect the digital works. The most promising solution for copyright protection of various multimedia contents is digital watermarking[2].

Digital watermarking refers to embedding a secret copyright holder identification data (logo, label, or a random sequence, etc) into the content of digital artworks (images, audio, video, etc) in the form of signals that are statistically undetectable and visually transparent. In general, the embedded data prepared for ownership verification or buyer identification[3][4]. Recently due to the enormous distribution of digital artworks via internet, the digital watermarking technology received more consideration [5].

In various literature, many watermarking techniques have been proposed by many authors over the past years. Some of them are simply implemented in the spatial domain while others utilize the major features of the transform domain[6]. In the spatial domain, watermarking techniques like Least Significant Bit (LSB), Spread Spectrum Modulation (SSM) and Quantization Index Modulation (QIM), the watermark bits are embedded directly into the raw data of the host signal without applying any transforms during the embedding process. On the other hand, in the transform domain, watermarking techniques like Discrete Fourier Transform (DFT), Discrete Cosine Transform (DCT) and Discrete Wavelet Transform (DWT), the host signal is firstly converted to corresponding transform domain representation then the watermark bits are embedded by modifying transform domain coefficients[7]. In fact, most current studies in the research areas of digital watermarking have been proposed in the transform domain due to its energy compaction capability which fulfils the imperceptibility property by spreading the changes introduced by the watermarks into the transform coefficients over all the pixels in the spatial domain[8] Generally, in order to be effective, the watermarking technique must satisfy the following basic properties[9]:

Unobtrusive: It means the embedded watermark should be invisible by the human eye, or it should not interfere with the digital content being protected.

Robust: It means the ability of watermark to resist common attacks, making it difficult to remove.

Unambiguous: It means the extracted watermark should identify the content owner unambiguously.

The motivation of this research work is to introduce a different application of transform domain based watermarking by utilizing the desired characteristics of DWT and SVD for the purpose of protecting the property rights of color artworks. In the extraction process, the proposed decoder requires side information to detect the presence of watermark. Accordingly, it can be used as a tool for developing a non-blind watermarking. The rest of the paper is structured as follows: Section 2 discusses the preliminaries required for this work. Section 3 describes in detail the proposed scheme. Section 4 includes the experimental results. Finally, the last section presents the concluding remarks and directions for future works.

\section{PRELIMINARIES}

As mentioned earlier, transform domain approach is presently more attractive and prevalent for watermarking than the spatial domain. This can be achieved by exploiting different transformation tools like DFT, DCT, SVD and DWT. The following subsections introduce a brief description of the DWT and SVD transformations.

\subsection{Discrete Wavelet Transform}

The DWT is an effective and useful approach for multi-resolution signal decomposition[10].In one-dimensional (1-D) signal applications, the DWT divides the signal in two parts: high frequency part and low frequency part. The high frequency part contains information about the edge components of the signal. While, the low frequency part is subdivided into two subsections of high and low frequency. This decomposition can continue until obtaining the desired level of signal decomposition[11]. In two-dimensional (2-D) signal applications, for each level of decomposition, the DWT is firstly performed in the vertical direction, followed by the DWT in the horizontal direction to produce four sub-bands namely: lower resolution approximation component (LL) as well as horizontal (HL), vertical (LH) and diagonal (HH) 
detail components[12][13]. Through the most counterpart studies, the best region for embedding the watermark is the mid-frequency sub-bands (HL and LH) in order to achieve a good trade-off between perceptual invisibility and robustness against various attacks[14][15]. Due to its special advantages over other conventional transforms, the DWT is currently used in a wide variety of signal processing applications such as audio and video compression, removing noise from audio signals, and the simulation of wireless antenna distribution[16][17][18][19].

\subsection{Singular Value Decomposition}

The SVD approach is one of the most familiar tools of linear algebra used in a big series of multimedia applications such as Image Compression, Watermarking and other Signal Processing[20]. The basic concept behind SVD approach is to decompose a rectangular matrix into three simple matrices: Two orthogonal matrices and one diagonal matrix[21]. Every real matrix A, can be broken down into a product of three matrices given by the following formula[22]:

$$
\mathrm{A}=\mathrm{USV}^{\mathrm{T}}
$$

Where,

$\mathrm{U}$ and $\mathrm{V}$ are the orthogonal matrices, $\mathrm{U}^{\mathrm{T}} \mathrm{U}=\mathrm{V}^{\mathrm{T}} \mathrm{V}=\mathrm{I}$, and $\mathrm{S}$ is the diagonal matrix, $S=\operatorname{diag}(\lambda 1, \lambda 2, \ldots \lambda r)$. The diagonal elements of $S$ are called the singular values of $A$, the columns of $\mathrm{U}$ are called the left singular vectors of A, and the columns of $\mathrm{V}$ are called the right singular vectors of $\mathrm{A}$.

In literature, SVD-based watermarking methods are classified into two types. In type 1, the watermark data is embedded directly into the singular values of the host signal. On the other hand, in type 2, the samples of the host signal are converted into their corresponding coefficients in the frequency domain then the watermark data is embedded in the singular coefficient values[23]. Actually, the popularity of SVD based watermarking depends on its remarkable properties, which mainly include [24][25]:

(I) Few number of singular values can represent large portion of signal energy.

(II) SVD can be performed into square and rectangular matrices.

(III) Good stability of SVs. This means that adding a small perturbation to host matrix, does not lead to great variance of its SVs.

(IV) SVs reflect the algebraic properties of host matrix, which are intrinsic rather than visual characteristics.

\section{PROPOSED ALGORITHM}

The suggested watermarking scheme consists of two process: watermark embedding and watermark extraction. The following subsections illustrate in detail the steps involved in each phase.

\subsection{Watermark Embedding Process}

The watermark embedding procedures are depicted in Figure. 1 and explained in the following steps:

Step 1: Read color artwork image as host and store it in I .

Step 2: Transform I from RGB color space into luminance and chrominance color space such as YCbCr. As the color representation in RGB is highly correlated, while the color representation in $\mathrm{YCbCr}$ is less correlated, the watermarking in $\mathrm{YCbCr}$ is more suitable .

$$
\begin{aligned}
& \text { If } \mathrm{Cb}_{\text {avg }}<\mathrm{Cr}_{\text {avg }} \\
& \text { Embedding Component }=\mathrm{Cb} \\
& \text { Ch_key }=2 \\
& \}
\end{aligned}
$$

Otherwise

\{

$$
\begin{aligned}
& \text { Embedding Component }=\mathrm{Cr} \\
& \text { Ch_key }=3 \\
& \}
\end{aligned}
$$

Step 3: Select the embedding component of chrominance part according to the color prevalence rate using the following formula:

Where,

Cbavg is the average value of blue chrominance component, Cravg is the average value of red chrominance component, Ch_key is the chrominance key which contains an integer number used as an indicator to determine any of the two chrominance components will be used in extraction process.

Step 4: Apply 1-level DWT using haar filters to decompose the selected embedding component $(\mathrm{Cb}$ or $\mathrm{Cr})$ into four sub-bands: LL1, HL1, LH1 and HH1.

Step 5: Select HL1 and apply SVD to it and obtain U1, S1 and V1.

Step 6: Read grayscale image as watermark and store it in W.

Step 7: Resize W to fit with the dimensions, width and height, of the selected embedding component.

Step 8: Apply 1-level DWT using haar filters to decompose W into four sub-bands: LL2, HL2, LH2 and HH2.

Step 9: Select HL2 and apply SVD to it and obtain U2, S2 and V2.

Step 10: Modify SVs of the HL1 with the SVs of HL2 using appropriate scaling factor as illustrated in the following formula:

$$
\mathrm{SW}=\mathrm{S} 1+\alpha . \mathrm{S} 2
$$

Where,

$\alpha$ is the scaling factor, which control the quality of the watermarked artwork image and the robustness of the extracted watermark.

Step 11: Construct the modified SVD matrix by U1, Sw, V1 and obtain HL1*.

Step 12: Apply inverse DWT to LL1, HL1 ${ }^{*}, \mathrm{LH} 1, \mathrm{HH} 1$ and obtain the modified chrominance component.

Step 13: Merge the luminance component and the two chrominance components and obtain the watermarked artwork image WI.

Step 14: Convert back WI from YCbCr color space to RGB color space. 
Step 15: Calculate the quality between the host artwork image I and the watermarked artwork image WI.

Step 16: Save the watermarked artwork image WI and the chrominance key Ch_key.

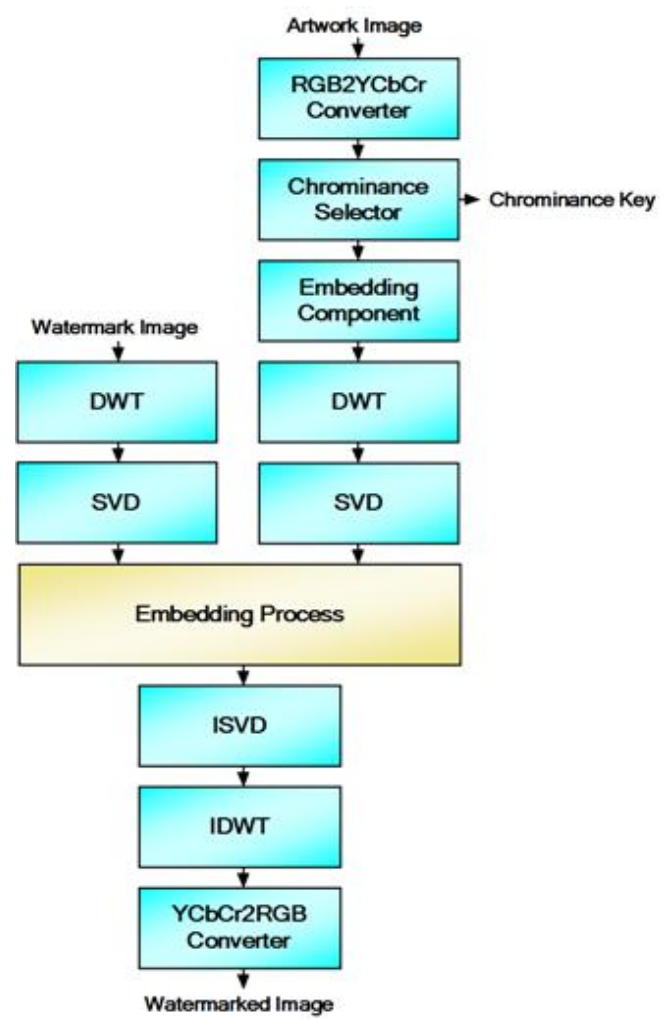

Fig 1: Structure of embedding process

\subsection{Watermark Extraction Process}

The watermark extraction procedures are performed in a reverse order to the embedding, as depicted in Figure.2. The details of the extraction process are described as follows:

Step 1: Read the watermarked version of color artwork image and store it in WI.

Step 2 Transform WI from RGB color space into $\mathrm{YCbCr}$ color space.

Step 3: Load the chrominance key and store it in Ch_key.

Step 4: Select the extraction component of chrominance part according to the value stored in chrominance key using the following formula:

$$
\text { If Ch_key }=2
$$$$
\{
$$$$
\text { Extraction Component }=\mathrm{Cb}
$$$$
\text { \} }
$$

\section{Otherwise}

\section{\{}

Extraction Component $=\mathrm{Cr}$

\section{\}}

Where,

Ch_key is the chrominance key generated in watermark embedding process.

Step 5: Apply 1-level DWT using haar filters to decompose the selected extraction component, $\mathrm{Cb}$ and $\mathrm{Cr}$, into four sub-bands: LL3, HL3, LH3 and HH3 .

Step 6: Select HL3 and apply SVD to it and obtain U3, S3 and V3.

Step 7: Read grayscale image as watermark and store it in W.

Step 8: Resize W to fit with the dimensions, width and height, of the selected extraction component.

Step 9: Apply 1-level DWT using haar filters to decompose W into four sub-bands: LL4, HL4, LH4 and HH4.

Step 10: Select HL4 and Apply SVD to it and obtain U4, S4 and V4.

Step 11: Extract singular values of watermark by using the following formula:

$$
\mathrm{Sw}^{*}=(\mathrm{S} 3-\mathrm{S} 4) / \alpha
$$

Where,

$\alpha$ is the same value of the scaling factor used in watermark embedding process.

Step 12: Construct the modified SVD matrix of watermark by $\mathrm{U} 4, \mathrm{Sw}^{*}, \mathrm{~V} 4$ and obtain $\mathrm{HL}^{*}$.

Step 13: Apply inverse DWT to LL4, HL4 ${ }^{*}, \mathrm{LH} 4, \mathrm{HH} 4$ and obtain the extracted watermark $\mathrm{W}^{*}$.

Step 14: Calculate the relation between the original watermark $\mathrm{W}$ and the extracted watermarked $\mathrm{W}^{*}$.

Step 15: Save the extracted watermark $\mathrm{W}^{*}$.

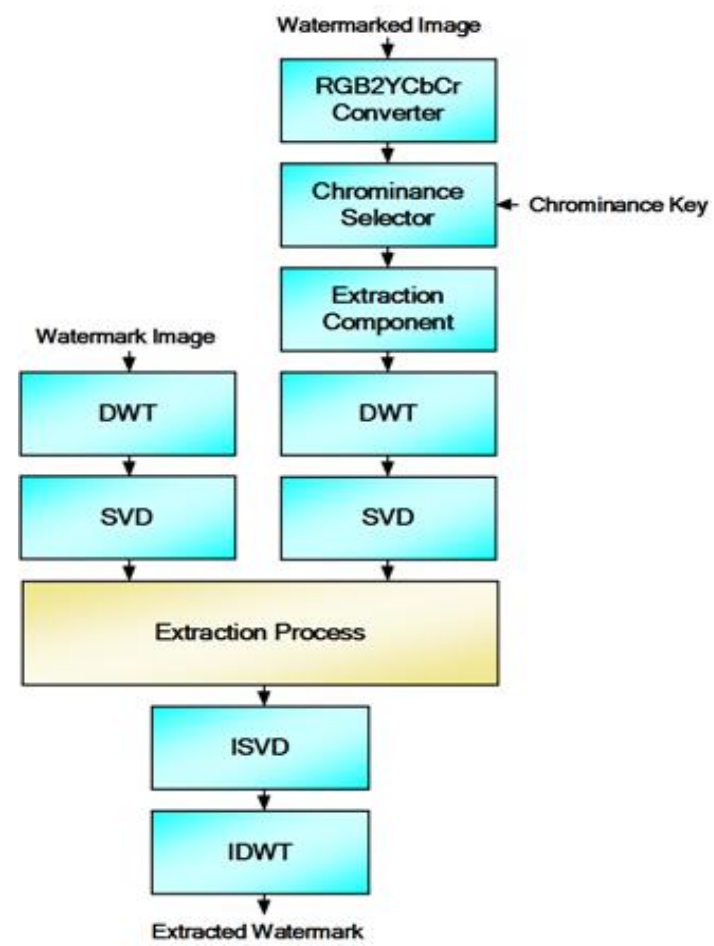

Fig 2: Structure of extraction process 


\section{EXPERIMENTSL RESULTS}

This section presents a complete analysis of the test scenario to experiment the proposed scheme. This scenario includes two parts: part 1 determines the experiment settings, which includes two subsections. The first subsection describes the test data and measurements, while the second subsection illustrates the strategy of selecting an optimal scaling factor. Part 2 evaluates the efficiency of the proposed scheme under different types of common attacks.

\subsection{Experiment Settings}

\subsubsection{Experimental Data and Measures}

For test evaluation purpose, a number of experiments using MATLAB simulation platform are done on two color artwork images with size $512 \times 512$, used as host and shown in Figure.3. The standard Grayscale image cameraman of size $256 \times 256$ taken as watermark is shown in Figure.4.
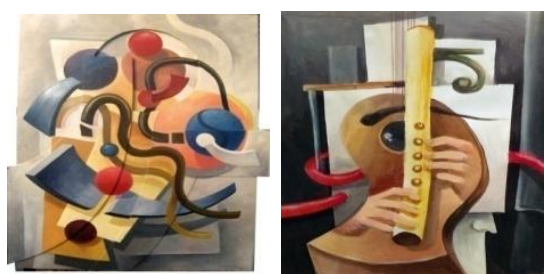

Fig.3: Host artwork images

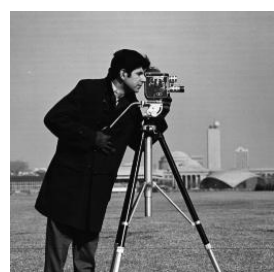

Fig.4: Watermark
To explore and analyze the performance of the proposed watermarking scheme, two evaluation metrics are used with reference to the most important requirements of the digital watermarking world: imperceptibility and robustness. The

first metric is peak signal to noise ratio(PSNR) which is used on a wide scale to measure the quality of the watermarked image regarded to the original one. PSNR in decibels $(\mathrm{dB})$ is given by the following equations[26].

$$
\begin{aligned}
\text { PSNR }_{\mathrm{dB}} & =10 \cdot \log 10\left(\frac{\mathrm{Max}^{2}}{\mathrm{MSE}}\right) \\
& =20 \cdot \log 10\left(\frac{\mathrm{Max}}{\sqrt{\mathrm{MSE}}}\right)
\end{aligned}
$$

Where,

Max represents the maximum possible pixel value in the image and MSE is the mean square error. In general, acceptable PSNR values range between 20 and $40 \mathrm{~dB}$. A good level of imperceptibility is obtained, if PSNR is more than 40 dB [ 27].

The second metric is Correlation Coefficient (Corr) which is utilized for measuring the relation degree between the original and the extracted watermark with or without performing digital attacks. Corr is given by the following equation[28].

$$
\text { Corr }=\frac{\sum_{i} \sum_{j}\left\{W(i, j)-W_{\text {avg }}\right\}\left\{W^{*}(i, j)-W^{*} \text { avg }\right\}}{\sqrt{\left\{\sum_{i} \sum_{j}\left[W(i, j)-W_{\text {avg }}\right]\right\}\left\{\sum_{i} \sum_{j}\left[W^{*}(i, j)-W^{*}{ }^{*}{ }^{2}\right]^{2}\right\}}}
$$

Where,

$\mathrm{W}$ is the original watermark, $\mathrm{W}^{*}$ is the extracted watermark. Generally, acceptable Corr is 0.75 or above[29][30].

\subsubsection{Selecting of Scaling Factor value a}

In our proposed watermarking scheme, the scaling factor influences the quality of the watermarked artwork image directly and obtains the best detection performance. In this context, the scaling factor should not exceed as it lessens the quality of watermarked artwork image (Imperceptibility) largely. In addition, it should not decline so as not to reduce the quality of extracted watermark (robustness) largely. For optimizing the scaling factor, several experiments are performed without applying any attacks upon watermarked artwork image to determine the relationship between the scaling factor and the two main watermarking parameters: PSNR and Corr. In these experiments all numerical results for each test image are calculated and the average scores of PSNR and Corr for the measured values of scaling factor are only recorded, as shown below in Table.1.

Table.1: Scaling factor versus PSNR and Corr

\begin{tabular}{|c|c|c|}
\hline \multirow{2}{*}{ Scaling factor } & \multicolumn{2}{|c|}{ Average } \\
\cline { 2 - 3 } & PSNR(I,WI) & Corr(W,W*) \\
\hline 0.1 & 51.35945 & 0.92875 \\
\hline 0.3 & 48.3536 & 0.99165 \\
\hline 0.5 & 46.24325 & 0.99695 \\
\hline 0.7 & 44.65355 & 0.99845 \\
\hline 0.9 & 43.402 & 0.999 \\
\hline
\end{tabular}

Based on the above mentioned results, increasing the scaling factor value helps in achieving a higher level of watermark recovery, but at the expense of the watermarked image quality. The obtained results indicate that there is a linear relationship between PSNR and the scaling factor, PSNR is inversely proportional to the scaling factor; the peak value of PSNR is when the scaling factor is equal to 0.1. Also the results indicate that there is a linear relationship between Corr and scaling factor, the Corr is directly proportional to the scaling factor; the peak value of Corr is when the scaling factor is equal to 0.9 . So there is a need for optimal selection of scaling factor to get a trade-off between imperceptibility and robustness. Considering these watermarking parameters, 0.5 is selected as the optimal scaling factor value for further experiments, since it provides the appropriate imperceptibility rate, average PSNR of 46.2432, and high level of robustness, average Corr of 0.9969 , in all test cases.

\subsection{Robustness Test}

To evaluate the performance of the proposed watermark detector against signal manipulations and degradations, different kinds of image processing attacks such as noise addition, JPEG compression, cropping, rescaling, filtering are applied to the watermarked artwork images. Samples of the visual effects of the aforementioned attacks on the watermarked artwork images and the extracted watermarks are shown in Figure.5, while all of these simulation results are recorded and listed in Table. 2 . 
Attacked artwork image

Extracted watermark
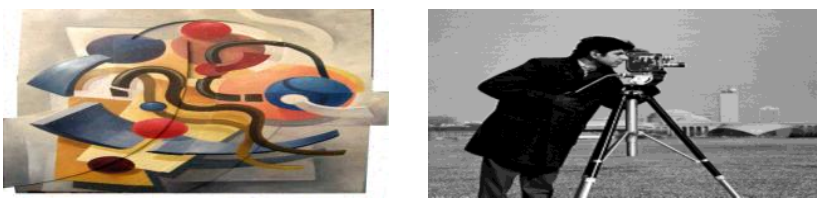

Salt \& pepper Noise (Density $=\mathbf{0 . 0 0 5})$
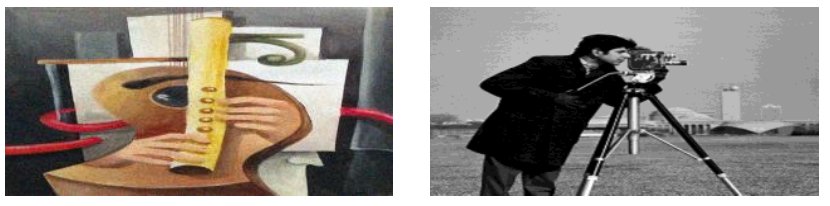

Gaussian Noise $($ Density $=\mathbf{0 . 0 5})$
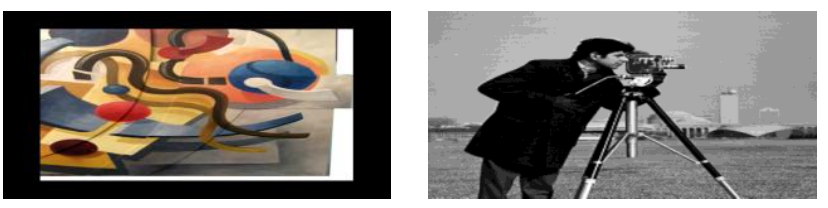

Cropping (Remaining Area=65\%).
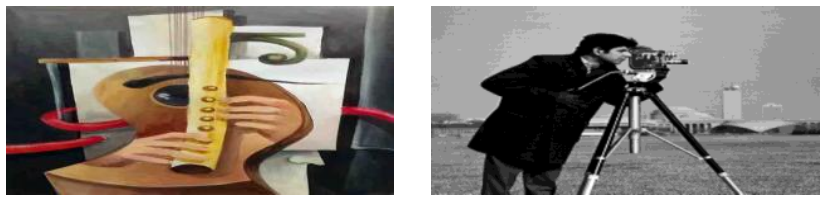

JPEG Compression $(\mathrm{QF}=25)$
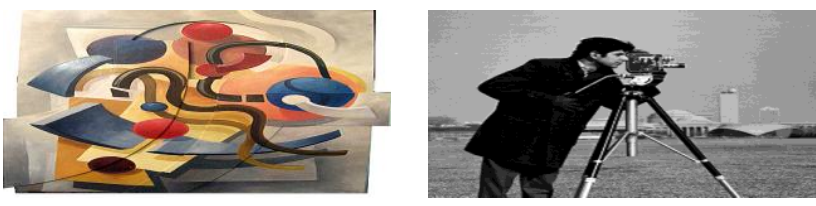

Sharpen Filter
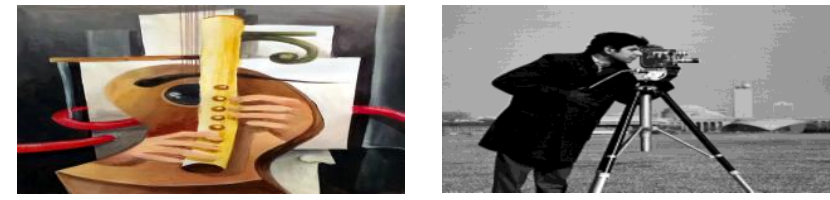

Intensity Adjustment
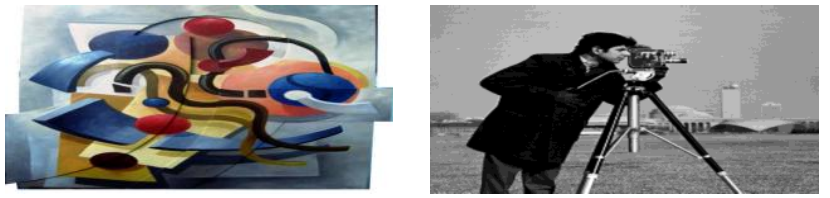

Histogram Equalization

Fig.5: Sample of test results
Table.2: Values of correlation coefficient obtained under common image attacks

\begin{tabular}{|c|c|c|c|}
\hline \multirow{2}{*}{ Attacks } & \multirow{2}{*}{ Parameter } & \multicolumn{2}{|c|}{$\operatorname{Corr}\left(\mathrm{W}, \mathrm{W}^{*}\right)$} \\
\hline & & $\begin{array}{l}\text { Attacked } \\
\text { artwork1 }\end{array}$ & $\begin{array}{l}\text { Attacked } \\
\text { artwork2 }\end{array}$ \\
\hline \multirow{2}{*}{$\begin{array}{c}\text { Salt \& pepper } \\
\text { Noise }\end{array}$} & Density $=0.005$ & 0.9985 & 0.9980 \\
\hline & Density $=0.05$ & 0.9866 & 0.9869 \\
\hline \multirow{2}{*}{$\begin{array}{c}\text { Gaussian } \\
\text { Noise }\end{array}$} & Density $=0.005$ & 0.9942 & 0.9944 \\
\hline & Density $=0.05$ & 0.9941 & 0.9942 \\
\hline \multirow{2}{*}{$\begin{array}{l}\text { Speckle } \\
\text { Noise }\end{array}$} & Density $=0.005$ & 0.9985 & 0.9976 \\
\hline & Density $=0.05$ & 0.9898 & 0.9951 \\
\hline \multirow{2}{*}{ Rotation } & Angle $=90$ & 0.9920 & 0.9895 \\
\hline & Angle $=180$ & 0.9978 & 0.9961 \\
\hline \multirow{2}{*}{ Flipping } & Dir=L2R & 0.9978 & 0.9961 \\
\hline & Dir $=U 2 D$ & 0.9978 & 0.9961 \\
\hline \multirow{2}{*}{ Rescaling } & $0.25 \gg>4$ & 0.9901 & 0.9888 \\
\hline & $0.5>>2$ & 0.9910 & 0.9893 \\
\hline Cropping & $\begin{array}{l}\text { Remaining } \\
\text { Area }=65 \%\end{array}$ & 0.9974 & 0.9952 \\
\hline $\begin{array}{c}\text { JPEG } \\
\text { Compression }\end{array}$ & $\mathrm{QF}=25$ & 0.9899 & 0.9886 \\
\hline $\begin{array}{l}\text { Sharpen } \\
\text { Filter }\end{array}$ & --- & 0.9881 & 0.9976 \\
\hline $\begin{array}{l}\text { Motion } \\
\text { Filter }\end{array}$ & --- & 0.9892 & 0.9882 \\
\hline $\begin{array}{l}\text { Blurring } \\
\text { Filter }\end{array}$ & --- & 0.9882 & 0.9876 \\
\hline $\begin{array}{l}\text { Average } \\
\text { Filter }\end{array}$ & --- & 0.9906 & 0.9889 \\
\hline $\begin{array}{c}\text { Intensity } \\
\text { Adjustment }\end{array}$ & --- & 0.9981 & 0.9969 \\
\hline $\begin{array}{c}\text { Histogram } \\
\text { Equalization }\end{array}$ & --- & 0.9987 & 0.9986 \\
\hline $\begin{array}{c}\text { Gamma } \\
\text { Correction }\end{array}$ & --- & 0.9982 & 0.9966 \\
\hline
\end{tabular}

From Figure.5, despite the different nature and effects of applicable attacks on the watermarked artwork image, the quality of extracted watermark is very fine and its details are clearly recognizable. In addition, all correlation scores recorded in Table. 2 are practically superior than 0.9 for all attacks, which well over the accepted threshold(described in section 4 part 1). These results mean that the performance achieved by the proposed scheme is promising and more robust against most applicable image processing attacks. 


\section{CONCLUSION}

This paper presents a digital watermarking scheme based different application of DWT and SVD for protecting color artworks. The proposed scheme extracts the watermark data with the help of the original watermark and the chrominance key, thus it belongs to a non-blind watermarking family. The simulation results on color artworks demonstrate that the proposed scheme is imperceptible and achieve a high level of robustness to most applicable attacks. In the future work, the focus will be on developing the proposed watermarking scheme to blind watermarking by reducing the necessities required in watermark extraction process.

\section{REFERENCES}

[1] Ta Minh, T., Munetoshi, I., "An Incomplete Cryptography based Digital Rights Management with DCFF", International Journal of Soft Computing and Software Engineering, Vol.3, No. 3, Special Issue: The Proceeding of International Conference on Soft Computing and Software Engineering, San Francisco State University, CA, U.S.A., PP: 507-513, 2013.

[2] Sharvari, C.T., Ratnadeep, R.D., "Blind 3D Model Watermarking based on Multi-Resolution Representation and Fuzzy Logic", International Journal of Computer Science \& Information Technology, Vol.4, No.1, PP: 117-126, 2012.

[3] Anitha, J., Immanuel, A.P.S., "A Color Image Digital Watermarking Scheme based on SOFM", International Journal of Computer Science, Vol. 7, No.5, PP: 302-309, 2010 .

[4] Solachidis, V., Tsekeridou, S., Nikolopoulos, S. and Pitas, I., "Self-Similar Watermarks for Counterfeiting Geometrical Attacks", in Proc. of 1st Wavila Challenge, Barcelona, Catalonia, Spain, 2005

[5] Mitesh, H.P., Swati, J.P., "2 ${ }^{\text {nd }}$ Level DWT based on Image Watermarking", International Journal of Engineering Development and Research, Vol.2, No.2, PP: 2789-2796, 2014.

[6] Chaw-Seng, W., "Digital Image Watermarking Methods for Copyright Protection and Authentication", A PhD Thesis Submitted to The Information Security Institute, Faculty of Information Technology, Queensland University of Technology, 2007.

[7] Radu, O.P., Cristina, O., Ionuț., P. and Lucian, A.P., "Robust Digital Video Watermarking in The Spatial and Wavelet Domain", The Seventh International Conference on Digital Telecommunications, PP:78-83, 2012.

[8] Zebbiche, K., Khelifi, F. and Bouridane, A., "An Efficient Watermarking Technique for the Protection of Fingerprint Images", Eurasip Journal on Information Security, PP:1-20, 2008.

[9] Thierry, B., Todor, T., "Improving the Watermarking Process with Usage of Block Error-Correcting Codes", Serdica Journal of Computing, Vol.2, No.2, PP: 163-180, 2008.

[10] Anand, K., Gite, B.B., "Secure Multimodal Authentication using Watermarking", International Journal of Innovative Research in Science, Engineering and Technology, Vol.3, Special Issue.4, PP: 215-219, 2014.
[11] Maklachur, R., "A DWT, DCT and SVD based Watermarking Technique to Protect the Image Piracy", International Journal of Managing Public Sector Information and Communication Technologies, Vol.4, No.2, PP: 21-32, 2013.

[12] Nikita, K., Sinha, G.R., "Image Watermarking using 3Level Discrete Wavelet Transform (DWT)", International Journal of Modern Education and Computer Science, , Vol.3, PP: 50-56, 2012.

[13] Anumol, J., Anusudha, K., "Robust Watermarking based on DWT SVD", International Journal of Signal \& Image Processing, Vol.1, No.1, 2013.

[14] Aparna, S.K., Sunita, S.L., "Imperceptible and Robust Digital Image Watermarking Techniques in Frequency Domain", International Journal of Computer Technology and Electronics Engineering, Vol.3, PP: 33-36, 2013.

[15] Amandeep, K., Blossom, K., Navneet, S., Sukhdeep, S. and Parneet, K., "Embedding Image in Mid Frequency Band using DWT Technique", International Journal of Advances in Engineering \& Technology, Vol.1, No.3, PP: 12-17, 2011.

[16] Asma, A., Sinha, G.R. and Nikita, K., "3-Level DWT Image Watermarking against Frequency and Geometrical Attacks", International Journal of Computer Network and Information Security, Vol.12, PP: 58-63, 2014.

[17] Hiral, L.B., Khyati, K.P., " Design and Implementation of Effective Architecture for Reversible Watermarking using FPGA", Journal of Emerging Technologies and Innovative Research, Vol.2, No.3, PP: 545-548, 2015.

[18] Prabhishek, S., Chadha, R.S., "A Survey of Digital Watermarking Techniques, Applications and Attacks", International Journal of Engineering and Innovative Technology, Vol.2, No.9, PP: 165-175, 2013.

[19] Jyoti, S., Dolley, S., "A Study Combined DWT-DCT Digital Image Hiding Information", International Journal of Computer Science and Information Technologies, Vol.6, No.3, PP: 2282-2286, 2015.

[20] Alexander, S., Scott, D. and Ahmet, M.E., "Robust DCTSVD Domain Image Watermarking for Copyright Protection: Embedding Data in All Frequencies", 13th European Signal Processing Conference, Antalya, Turkey, 2005.

[21] Swanirbhar, M., Madhusudhan, M. and Aheibam, D.S. "A Hybrid SVD and Wavelet based Watermarking", $2^{\text {nd }}$ National Conference Mathematical Techniques: Emerging Paradigms for Electronics and IT Industries, PP: 197-201, 2008.

[22] Rajani, A., Ramashri, T., "Image Watermarking Algorithm using DCT, SVD and Edge Detection Technique", International Journal of Engineering Research and Applications, Vol.1, No.4, PP: 1828-1834.

[23] Bokan, O.A., Ghazali, B.S., "Semi-Blind RGB Color Image Watermarking using DCT and Two-Level SVD", International Journal of Signal \& Image Processing, Vol.4, No.5, PP:1-10, 2013.

[24] Kala, A., Thaiyalnayaki, K., "Robust Lossless Image Watermarking in Integer Wavelet Domain using SVD", International Journal of Computer Science Engineering, Vol.2, No.02, PP: 30-35, 2013. 
[25] Nilesh, R., Ganga, H., "Securing Medical Images by Watermarking using DWT-DCT-SVD", International Journal of Computer Trends and Technology, Vol.X, No.Y, PP: 1-9, 2014

[26] Ali, A., "Combined DWT-DCT Digital Image Watermarking", Journal of Computer Science, Vol.3, No.9, PP: 740-746, 2007.

[27] Deepak, A., Kanwalvir, S.D., "Effect of Embedding Watermark on Compression of the Digital Images", Journal of Computing, Vol.2, No.2, PP:5-8, 2010.

[28] Afroja, A., Muhammad, A.U., "Digital Watermarking with A New Algorithm", International Journal of
Research in Engineering and Technology, Vol.03, No.03, PP: 212-217, 2014.

[29] Neelima, K.B., Saravanan, T., "Sample Image Watermarking Study", International Journal of Multidisciplinary Research and Development, Vol.2, No.5, PP: 90-92, 2015.

[30] Muhammad, S.S., Yasuhiko, D., "A Watermarking Scheme for Digital Images using Multilevel Wavelet Decomposition", Malaysian Journal of Computer Science, Vol.16, No.1, PP: 24-36, 2003. 